\title{
How soon to start: aspirin resumption after upper gastrointestinal bleed?
}

\author{
Rebecca Gooch and Marie Baldisseri* \\ University of Pittsburgh Department of Critical Care Medicine: Evidence-Based Medicine Journal Club, edited by Sachin Yende
}

\section{Extended abstract \\ Citation \\ Continuation of low-dose aspirin therapy in peptic ulcer bleeding: a randomized trial. Sung JJ, Lau JY, Ching JY, Wu JC, Lee YT, Chiu PW, Leung VK, Wong VW, Chan FK. Ann Intern Med. 2010 Jan 5;152(1):1-9. Epub 2009 Nov 30.}

\section{Background}

It is uncertain whether aspirin therapy should be continued after endoscopic hemostatic therapy in patients who develop peptic ulcer bleeding while receiving lowdose aspirin.

\section{Objective}

To test that continuing aspirin therapy with protonpump inhibitors after endoscopic control of ulcer bleeding was not inferior to stopping aspirin therapy, in terms of recurrent ulcer bleeding in adults with cardiovascular or cerebrovascular diseases.

\section{Design}

A parallel randomized, placebo-controlled noninferiority trial, in which both patients and clinicians were blinded to treatment assignment, was conducted from 2003 to 2006 by using computer-generated numbers in concealed envelopes. (ClinicalTrials.gov registration number: NCT00153725)

\section{Setting}

A tertiary endoscopy center.

\section{Patients}

Low-dose aspirin recipients with peptic ulcer bleeding.

\section{Intervention}

78 patients received aspirin, $80 \mathrm{mg} / \mathrm{d}$, and 78 received placebo for 8 weeks immediately after endoscopic

\footnotetext{
*Correspondence: baldisserimr@upmc.edu

'Department of Critical Care Medicine, University of Pittsburgh, 614 Scaife Hall, 3550 Terrace Street, Pittsburgh, PA 15261, USA
}

therapy. All patients received a 72-hour infusion of pantoprazole followed by oral pantoprazole. All patients completed follow-up.

\section{Measurements}

The primary end point was recurrent ulcer bleeding within 30 days confirmed by endoscopy. Secondary end points were all-cause and cause-specific mortality in 8 weeks.

\section{Results}

156 patients were included in an intention-to-treat analysis. Three patients withdrew from the trial before finishing follow-up. Recurrent ulcer bleeding within 30 days was $10.3 \%$ in the aspirin group and $5.4 \%$ in the placebo group (difference, 4.9 percentage points [95\% CI, -3.6 to 13.4 percentage points]). Patients who received aspirin had lower all-cause mortality rates than patients who received placebo ( $1.3 \%$ vs. $12.9 \%$; difference, 11.6 percentage points [CI, 3.7 to 19.5 percentage points]). Patients in the aspirin group had lower mortality rates attributable to cardiovascular, cerebrovascular, or gastrointestinal complications than patients in the placebo group (1.3\% vs. $10.3 \%$; difference, 9 percentage points [CI, 1.7 to 16.3 percentage points]).

\section{Limitations}

The sample size is relatively small, and only low-dose aspirin, $80 \mathrm{mg}$, was used. Two patients with recurrent bleeding in the placebo group did not have further endoscopy.

\section{Conclusion}

Among low-dose aspirin recipients who had peptic ulcer bleeding, continuous aspirin therapy may increase the risk for recurrent bleeding but potentially reduces mortality rates. Larger trials are needed to confirm these findings.

\section{Commentary}

Fifty million Americans use low-dose aspirin, 325mg/day or less, regularly for cardioprophylaxis ${ }^{1}$. The estimated 
average excess risk of upper gastrointestinal bleeding (UGIB) related to cardioprophylactic doses of ASA is 5 cases per 1000 ASA users per year ${ }^{2}$. Currently the 2010 International Consensus on Non-variceal Upper Gastrointestinal Bleeding is that - In patients who receive lowdose ASA and develop acute ulcer bleeding, ASA therapy should be restarted as soon as the risk for cardiovascular complication is thought to outweigh the risk for bleeding. ${ }^{3}$ One significant problem with this recommendation is that the risks of when one outweighs the other is very ill defined. It has been shown that prolonged discontinuation of ASA therapy increases thrombotic risk ${ }^{4}$. This creates a conundrum for physicians trying to balance the risk of re-bleed vs. cardiac or cerebrovascular event risk.

The purpose of this study was to determine that restarting ASA therapy after endoscopic control of the UGIB was not inferior to stopping ASA therapy. It was a randomized placebo non-inferiority study where patients were randomly assigned to placebo or ASA therapy after endoscopic control of an UGIB. The primary outcome was to evaluate the occurrence of recurrent peptic ulcer bleed within 30 days of the initial event. Secondary outcomes included all cause mortality, and death attributed to cardiovascular, cerebrovascular or gastrointestinal complications. Secondary endpoints included blood transfusion requirement, duration of hospital stay, requirement of surgery, and recurrence of acute ischemic events (ACS/CVA). There was no significant difference in the primary outcome measure, incidence of recurrent ulcer bleeding at 30 days $(10.3 \%$ in the low-dose aspirin group and $5.4 \%$ in the placebo group, difference 4.9 percentage points, [CI -3.6 to 13.4 percentage points]). All cause mortality was lower in the ASA group (1.3\% in the ASA group at 56 days and $12.9 \%$ in the placebo group, difference of 11.6 percentage points, [CI 3.7 to 19.5 percentage points]). There was no difference between the two groups regarding the other secondary outcomes.

The strength of this paper is that it was a well designed study. The patients were well randomized and the evaluators were blinded to the treatment groups. Limitations include a small sample size and concerns as to whether the information can be extrapolated to patients that use higher doses of ASA. In addition, there were 2 patients in the placebo group that did not undergo endoscopy -1 died before getting to the hospital, and the other patient was too unstable for recurrent endoscopy. These two patients were not added to the primary analyses. If they were added to the cases of recurrent bleeding the difference between the two groups would be reduced.

The findings of this study suggest that early resumption of ASA has a trade-off. While early resumption increased risk of re-bleeding, it reduced risk of subsequent cardiovascular events. This study used a single primary endpoint and non-inferiority margin of $10 \%$, i.e. the trial had adequate power to detect a $>10 \%$ higher risk of rebleeding in the group where ASA was resumed immediately. For instance, the re-bleeding risk in the group where ASA was resumed immediately was $4.9 \%$ higher compared to the group in whom ASA was resumed later. This difference was not statistically significant, but the study was underpowered to detect such small differences. These findings raise questions regarding the optimal design of trials to address this issue. Is the $10 \%$ noninferiority margin acceptable and should the authors have designed a study to detect smaller differences in rebleeding risk? Similar to prior studies, ${ }^{5}$ one approach would be to use a non-inferiority design to compare bleeding risk and superiority design to compare all-cause mortality.

The results of this study need to be confirmed in a larger study as its treatment implications for all health providers could be great in terms of managing their patients after UGI bleeds. Like any good study, it raises several additional questions: What is the appropriate dose of ASA that should be restarted? What is the appropriate time interval from endoscopy until ASA resumption?

\section{Recommendation}

In conclusion, patients that have an UGIB that need cardioprophylaxis or cerebrovascular prophylaxis should be restarted on their ASA therapy as soon as endoscopic control of their UGIB source has been controlled.

\section{Competing interests}

The authors declare that they have no competing interests.

\section{Published: 20 December 2010}

\section{References}

1. Chan FK, Graham DY: Prevention of non-steroidal anti-inflammatory drug gastrointestinal complications - review and recommendations based on risk assessment. Aliment Pharmacol Ther 2004, 19:1051-61.

2. Weil J, Colin-Jones D, Langman M, Lawson D, Logan R, Murphy M, Rawlins M, Vessey $M$, Wainwright P: Prophylactic aspirin and risk of peptic ulcer bleeding. BMJ 1995, 310:827-30.

3. Barkun AN, Bardou M, Kuipers EJ, Sung J, Hunt RH, Martel M, Sinclair P; International Consensus Upper Gastrointestinal Bleeding Conference Group: International consensus recommendations on the management of patients with nonvariceal upper gastrointestinal bleeding. Ann Intern Med 2010, 152:101-113.

4. Aguejouf O, Eizayaga F, Desplat V, Belon P, Doutremepuich C: Prothrombotic and hemorrhagic effects of aspirin. Clin App/ Thromb Hemost 2009, 15:523-8

5. Bouadma L, Luyt CE, Tubach F, Cracco C, Alvarez A, Schwebel C, Schortgen F, Lasocki S, Veber B, Dehoux M, Bernard M, Pasquet B, Régnier B, Brun-Buisson C, Chastre J, Wolff M; PRORATA trial group: Use of procalcitonin to reduce patients' exposure to antibiotics in intensive care units (PRORATA trial): a multicentre randomized controlled trial. Lancet 2010, 375:463-474.

doi:10.1186/cc9368

Cite this article as: Gooch R, Baldisseri M: How soon to start: aspirin resumption after upper gastrointestinal bleed? Critical Care 2010, 14:331. 